\title{
Status of the Forward Physics Projects in ATLAS
}

\author{
Stefan Ask $^{1}$ \\ (on behalf of the ATLAS Luminosity and Forward Physics Working Group) \\ 1- CERN - Physics Department \\ CH-1211 Geneva 23 - Switzerland
}

\begin{abstract}
The ATLAS experiment at the LHC is building several detector systems for forward physics studies and to determine the luminosity. The main forward systems consist of a Cerenkov detector called LUCID, a Zero Degree Calorimeter (ZDC) and Roman Pots which will house a scintillating fiber tracker system called ALFA. Here we report some of the forward physics activities that are foreseen in ATLAS together with the status of the related detector systems.
\end{abstract}

\section{Forward detectors in ATLAS}

In addition to the main ATLAS detector, also three smaller systems are built to cover the forward region [2]. These are closely connected to the luminosity determination in ATLAS, but are in addition foreseen to study forward physics. When ordered by their distance from the ATLAS interaction point (IP) the first system is a Cerenkov detector called LUCID. LUCID is the main luminosity monitor in ATLAS and is located $17 \mathrm{~m}$ away from the IP. The second system is the so-called zero degree calorimeter (ZDC) which is located at a distance of $140 \mathrm{~m}$ from the IP. This corresponds to the location where the LHC beam-pipe is divided into two and the ZDC is located between the beam pipes just after the split inside the so-called TAN absorber. The most remote system is the so-called ALFA system. ALFA consists of scintillating fiber trackers located inside roman pots at a distance of $240 \mathrm{~m}$ from the ATLAS IP. All results presented below are preliminary.

ATLAS also foresee upgrades of the roman pot program with stations at $220 \mathrm{~m}$ and $420 \mathrm{~m}$ dedicated entirely to diffractive physics, however, the status of these projects are presented by C. Royon [3] and A. Pilkington [4] at this conference.

\section{The ALFA system}

The ALFA (Absolute Luminosity For ATLAS) system consists of scintillating fiber trackers located in roman pots at a distance of $240 \mathrm{~m}$ on each side of the IP. The roman pots allow the detectors to approach the beam inside the LHC beam-pipe and the main purpose of ALFA is to measure elastic proton scattering at low angles. This is primarily to determine the absolute luminosity in ATLAS, but also other physics studies are foreseen such as measuring the total $p p$ cross section, measuring elastic scattering parameters and potentially also to tag protons for diffractive studies.

For a maximum precision in the luminosity measurement, the goal is to measure elastic scattering in the Coulomb interference region, which requires a measurement of scattering angles down to about $3 \mu \mathrm{rad}$. In order to reach such small angles, the LHC has to run with special so-called high $\beta^{*}$ optics, but even with this optics the detectors have to be located at a distance of only $1-2 \mathrm{~mm}$ from the beam. The main requirements on the tracker are, a spatial resolution of about $30 \mu m$, no significant non-active edge region, insensitivity to 
the $\mathrm{RF}$ from the LHC beam and to the vacuum in the roman pot. The high $\beta^{*}$ runs have a very low luminosity and for this reason no radiation hard technology have to be adopted.

Due to these requirements, ATLAS has chosen a scintillating fiber tracker. Prototype detectors of the ALFA tracker have been validated in beam tests at DESY [5] and CERN together with the front-end electronics and the so-called overlap detector alignment system. The tests have shown an adequate performance for the luminosity measurement and the full ALFA system is foreseen to be installed in the shutdown between 2008 and 2009.

In parallel to the detector development, the measurement of elastic scattering have been carefully simulated. The primary analysis is based on a fit of the differential cross section of elastic scattering (simplified below),

$$
\begin{gathered}
\frac{d N}{d t}=L \cdot \pi\left|-\frac{2 \alpha}{|t|}+\frac{\sigma_{t o t}}{4 \pi}(i+\rho) e^{-B|t| / 2}\right|^{2} \\
t=-(p \cdot \sin \theta)^{2}
\end{gathered}
$$

to the $t$-distribution of the data. Figure 1 shows the reconstructed $t$-distribution from simulations of the ALFA measurement. As seen in the plot, the acceptance covers the interference region where the EM contribution becomes significant and give rise to the steeper slope at low $t$-values. Several sys-

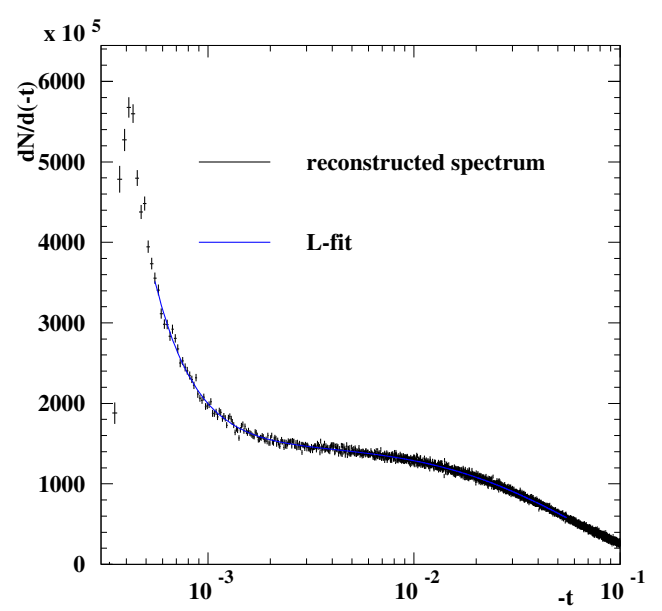

Figure 1: Simulation of the reconstructed $|t|-$ distribution from the ALFA measurement. tematic errors have been studied, for example due to beam properties, detector acceptance, alignment and background. The precision of the luminosity measurement from the fit is estimated to be $\mathcal{L} \pm 2 \%$ (stat) $\pm 2 \%$ (syst). Also alternative methods to determine the luminosity are foreseen such as using the optical theorem.

The fit allows a measurement of the total $p p$ cross section $\left(\sigma_{t o t}\right)$, the nuclear slope parameter $(B)$ and the ratio of the real and imaginary part of the nuclear amplitude $(\rho)$. The current results only includes statistical errors, but these indicates that the listed parameters will be possible to measure with a precision of the order of $1 \%, 0.5 \%$ and $4 \%$ respectivelly.

\section{The LUCID system}

LUCID (LUminosity measurement using Cerenkov Integrating Detector) is the main luminosity monitor in ATLAS. Its main purpose is to detect inelastic $p p$ scattering in the forward direction, both in order to measure the integrated luminosity of the ATLAS runs and for on-line monitoring of the instantaneous luminosity and beam conditions. Potentially LUCID could also be used for diffractive studies, e.g. as a rapidity gap veto.

The luminosity monitoring is based on the fact that the inelastic $p p$ rate $\left(R_{p p}\right)$ seen by LUCID is proportional to the luminosity,

$$
R_{p p}=\mu_{L U C I D} \cdot f_{B C}=\sigma_{\text {inel }} \cdot \varepsilon_{L U C I D} \cdot \mathcal{L}
$$


Here the mean number of inelastic $p p$ interactions per bunch crossing (BC) seen by LUCID, $\mu_{L U C I D}$, is related to the luminosity $(L)$ by the inelastic cross section $\left(\sigma_{\text {inel }}\right)$ and the LUCID detection efficiency $\left(\varepsilon_{L U C I D}\right)$. In this equation $f_{B C}$ represent the bunch crossing rate. The value of $\mu_{L U C I D}$ can be measured by LUCID in several ways [6],

- Zero Counting:

- Hit Counting:

- Particle Counting:

$$
\begin{aligned}
& \mu_{L U C I D}=-\ln \left(N_{\text {ZeroBC }} / N_{\text {TotBC }}\right) \\
& \mu_{\text {LUCID }}=\left\langle N_{\text {Hits } / B C}\right\rangle /\left\langle N_{\text {Hits } / \text { pp }}\right\rangle \\
& \mu_{\text {LUCID }}=\left\langle N_{\text {Particles } / B C}\right\rangle /\left\langle N_{\text {Particles } / \text { pp }}\right\rangle .
\end{aligned}
$$

The first method determines $\mu_{L U C I D}$ from the ratio between the number of non-colliding $\mathrm{BCs}$ and the total number of BCs. The two following methods in principle determine $\mu_{L U C I D}$ from the ratio of the mean number of particles per $\mathrm{BC}$ and the mean number of particles per inelastic interaction, both seen by LUCID. Hit counting normally refers to particle counting, but where the counting capability of the detector is limited by its granularity.

The main requirements of the corresponding detector system are, an acceptance to minimum bias events, sufficient time resolution to measure individual BCs and being capable of counting particles. For this purpose ATLAS has chosen the LUCID detector which consists of aluminum tubes filled with $C_{4} F_{10}$ surrounding the beam-pipe and which are pointing at the ATLAS IP. The Cerenkov light emitted by a transversing particle is reflected down the tube and read-out by PMTs. The signal amplitude from the PMTs can be used to distinguish the number of particles per tube and the fast time response allows to measure individual BCs. A small scale LUCID, dedicated purely to luminosity monitoring, has been validated in testbeams and will be installed for the start up of the LHC. Based on the performance of the initial detector an optimized upgrade, including a large number of tubes, is foreseen to be installed at the same time as the upgrade of the LHC for the nominal luminosity of $\mathcal{L}=10^{34} \mathrm{~cm}^{-2} \mathrm{~s}^{-1}$.

For the luminosity measurement, the general calibration strategy of LUCID is to run in parallel with an absolute measurement of the luminosity at the ATLAS IP. Initially this will most likely be obtained from the LHC machine parameters with an expected precision of about $10-15 \%$. This will hopefully be improved in the medium term by studies of well known physics processes, like for example $W$ or $Z$ production as discussed in [7] at this conference. When the ALFA measurement is available this will be the main reference for calibration. In this scenario the parallel measurement of $\mu_{L U C I D}$ and $L$ will be made at optimal conditions for the absolute method (which provides $L$ ). The calibration constant, containing $\sigma_{\text {inel }}$ and $\varepsilon_{L U C I D}$, can then be determined, allowing the LUCID measurement to directly provide the luminosity at different conditions.

\section{The ZDC system}

The third forward system in ATLAS is the zero degree calorimeter, which will measure neutral particles at a $0^{\circ}$ polar angle. The ZDC has a central role in the ATLAS heavy ion (HI) program where it is used to measure the centrality of the collisions, the luminosity as well as to provide triggers. It will, however, also be of importance both in the $p p$ program as described below and for accelerator tuning where it can be used to determine the location of the IP and the beam crossing angle.

The ATLAS ZDC consists of six tungsten/quartz calorimeter modules where the light from the quartz fibers is read-out by PMTs. In addition the ZDC is equipped with horizontal quartz rods, parallel to the beam, in order to determine the location of the showers in the 
plane perpendicular to the beam. The ZDC has been extensively tested and will be installed at the start up of the LHC. An upgrade is foreseen after about one year of running when additional space in the TAN absorber will become available.

In the HI runs the main purpose of the ZDC is to measure the spectator neutrons. These are remnants of the collision and provides information about both the magnitude and direction of the impact parameter. In addition, the ZDC have close to a $100 \%$ acceptance for HI collisions and together with the well known cross section of neutral particles at a zero degree angle the luminosity can be determined to a precision better than 5\%. It was also shown at RHIC that neutron tagging with the ZDC was essential to de-

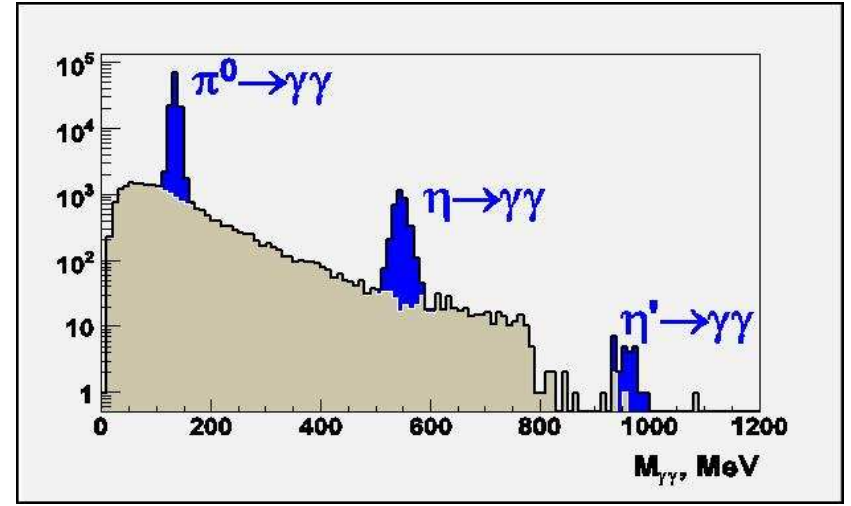

Figure 2: Simulated invariant mass spectrum as measured by the ZDC. sign a low rate trigger for ultraperipheral events.

In the ATLAS $p p$ program the ZDC will mainly be used to study forward particle production. Figure 2 shows a simulated invariant mass spectrum as measured by the ZDC. Several meson peaks are clearly visible and also other mesons and baryons can be reconstructed. The cross section measurements of particles in the forward direction at the LHC energy is of interest for several applications. For example the measurement is of large interest to the high energy cosmic ray community where the information is required to properly model air showers from high energy protons entering the atmosphere, where the proton energy at the LHC, $E_{l a b}=10^{17} \mathrm{eV}$, is just below the knee in the cosmic ray energy spectrum. In addition the ZDC will add to the overall hermeticity of ATLAS which will be useful to suppress background in diffractive studies.

\section{References}

[1] Slides: http: //indico. cern.ch/contributionDisplay. py? contribId=97\&sessionId=7\&conf Id=9499

[2] ATLAS Forward Detectors for Measurement of Elastic Scattering and Luminosity Determination, ATLAS TDR, in preparation;

ATLAS Forward Detectors for Luminosity Measurement and Monitoring, CERN-LHCC/04-10, LHCC $\mathrm{I}-014$

Zero Degree Calorimeters for ATLAS, CERN-LHCC/07-01, LHCC I-016.

[3] High mass diffractive physics at the LHC, C. Royon at this conference.

[4] Status of FP420 RED project, A. Pilkington at this conference.

[5] S. Ask et al., Nucl. Instr. and Meth. A 568 (2006) 588-600.

[6] S. Ask, ATL-LUM-PUB-2006-001.

[7] Precision PDF measurements at the LHC, A. Cooper-Sarkar at this conference. 